\title{
Crystal Structure of Human DNA Methyltransferase 1
}

\author{
Zhi-Min Zhang ${ }^{1}$, Shuo Liu ${ }^{2,3}$, Krystal Lin ${ }^{1}$, Youfu Luo ${ }^{4}$ John Jefferson Perry ${ }^{1}$, Yinsheng Wang ${ }^{2,3}$, Jikui \\ Song ${ }^{1}$ \\ ${ }^{1}$ Department of Biochemistry, University of California, Riverside, CA 92521, USA. \\ ${ }^{2}$ Environmental Toxicology Graduate Program, University of California, Riverside, CA 92521, USA. \\ ${ }^{3}$ Department of Chemistry, University of California, Riverside, CA 92521, USA. \\ ${ }^{4}$ State Key Laboratory of Biotherapy/Collaborative Innovation Center for Biotherapy, West China \\ Hospital, West China Medical School, Sichuan University, Chengdu, Sichuan 610041, PR China. \\ *Keyword: DNA methylation, DNMT1, Epigenetics, allosteric regulation, autoinhibition \\ To whom correspondence should be addressed: Jikui Song, Department of Biochemistry, University of \\ California, Riverside, CA 92521, USA, Tel: +1 951827 4221; Fax: +1 951827 4294; Email: \\ jikui.song@ucr.edu
}

\begin{abstract}
DNA methyltransferase 1 (DNMT1) is responsible for propagating the DNA methylation patterns during DNA replication. DNMT1 contains, in addition to a C-terminal methyltransferase domain, a large Nterminal regulatory region that is comprised of an RFTS (replication foci targeting sequence) domain, a CXXC zinc finger domain and a pair of BAH (bromo adjacent homology) domains. The regulatory domains of DNMT1 mediate a network of protein-protein and protein-DNA interactions to control the recruitment and enzymatic activity of DNMT1. Here we report the crystal structure of human DNMT1 with all the structural domains (hDNMT1, residues 351-1600) in complex with $S$-adenosyl-Lhomocysteine at $2.62 \AA$ resolution. The RFTS domain directly associates with the methyltransferase domain, thereby inhibiting the substrate binding of hDNMT1. Through structural analysis, mutational, biochemical and enzymatic studies, we further identify that a linker sequence between the CXXC and BAH1 domains, aside from its role in the CXXC domain-mediated DNMT1 autoinhibition, serves as an important regulatory element in the RFTS domain-mediated autoinhibition. In comparison with the previously determined structure of mouse DNMT1, this study also reveals a number of distinct structural
\end{abstract}


features that may underlie subtle functional diversity observed for the two orthologues. In addition, this structure provides a framework for understanding the functional consequence of disease-related hDNMT1 mutations.

\section{Introduction}

DNA methylation is one of the key epigenetic mechanisms that are essential for transcriptional silencing of retrotransposons (1-3), genomic imprinting (4), and X-chromosome inactivation (5). DNA methylation in mammals mainly occurs at the C-5 position of cytosine within the symmetric CpG dinucleotide and affects $\sim 70-80 \%$ of the $\mathrm{CpG}$ dinucleotides throughout the genome (6). The DNA methylation patterns are established by the de novo DNA methyltransferases DNMT3A and DNMT3B during gametogenesis and early embryogenesis, and become stably inherited by the maintenance DNA methyltransferase DNMT1, in cooperation with DNMT3A and DNMT3B (7-10). DNMT1-mediated maintenance DNA methylation is supported by both its substrate preference toward hemimethylated CpG sites $(11,12)$, and its recruitment to DNA replication foci $(13,14)$, through its interactions with proliferating cell nuclear antigen (PCNA) (15) and histone H3 ubiquitinated at lysine 23 (16).

DNMT1 is a multi-modular protein that is comprised of $\sim 1,620$ amino acids. It contains a C-terminal methyltransferase (MTase) domain and a large N-terminal regulatory region, linked by a conserved (GK)n dipeptide repeat. The N-terminal region of DNMT1 is composed of an RFTS (replication foci targeting sequence) domain, a CXXC zinc finger domain, and a pair of BAH (bromo adjacent homology) domains. These N-terminal domains distinguish DNMT1 from its bacterial counterparts and impose a tight control on the recruitment and enzymatic activity of DNMT1 $(7,17)$. To elucidate the regulatory mechanisms of DNMT1, we have previously determined the crystal structures of a C-terminal fragment of mouse DNMT1 (residues 650-1602, mDNMT1 $_{650-1602}$ ) in complex with an unmethylated CpG DNA, and the equivalent human DNMT1 ( DDNMT1 $_{646-1600}$ )-DNA complex, at $3.0 \AA$ and $3.6 \AA$ resolution, respectively (18). These structures reveal that the DNMT1 CXXC domain specifically binds to the CpG 
dinucleotide, which helps position the CXXC-BAH1 domain linker (a.k.a. autoinhibitory linker) into the catalytic cleft of the MTase domain, thereby forming an autoinhibitory conformation. This observation, together with mutational studies and enzymatic activity assays, suggests that the DNMT1 CXXC domain plays an inhibitory role in DNMT1-mediated de novo methylation. In a separate study, Takeshita et al (19) has determined the structure of a longer mDNMT1 fragment (residues 291-1620, mDNMT1 $_{291-1620}$ ), free and in complex with cofactor $S$-adenosyl-L-methionine (AdoMet) or cofactor product $S$-adenosyl-Lhomocysteine (AdoHcy). The structure of $\mathrm{mDNMT1}_{291-1620}$ reveals that the RFTS domain forms a DNA competitive inhibitor through direct interaction with the MTase domain of mDNMT1. This observation suggests that the RFTS domain plays an inhibitory role in DNMT1-mediated methylation, and has subsequently been supported by a number of biochemical and cellular studies (19-22).

Human DNMT1 and its mouse orthologue share about $80 \%$ sequence identity, which raises a question about whether the structure of mDNMT1 can fully recapitulate the functional state of hDNMT1. Indeed, previous enzymatic analysis of full-length recombinant hDNMT1 and mDNMT1 proteins over the poly (dI-dC).poly(dI-dC) substrates have revealed a 30-fold difference in their $k_{\text {cat }}$ values (23-25). Our recent studies have also shown that mDNMT1 $_{650-1602}$ and its human counterpart, hDNMT1 $1_{646-1600}$, methylate a 14-mer hemimethylated CpG DNA with 2-3 fold different $k_{\text {cat }}$ and $K_{\mathrm{m}}$ values (18). Together, these observations suggest that subtle functional diversity may exist between the two orthologues. In addition, the relative low resolution $(2.75-3.25 \AA)$ of the structure of mDNMT $_{291-1620}$ limited detailed analysis of protein interactions, such as the water-mediated hydrogen bonding. In this study, we report the crystal structure of a long C-terminal fragment of hDNMT1 (residues 351-1600, hDNMT1 ${ }_{351-1600}$ ) in complex with AdoHcy at $2.62 \AA$ resolution. This structural study, combined with mutational, biochemical and enzymatic analysis, provides deep insights into the allosteric regulation of hDNMT1 and its structurefunction diversity from mDNMT1. Importantly, this study reveals that the DNMT1 CXXC-BAH1 domain linker plays an important role in two different autoinhibitory mechanisms of DNMT1. It also 
provides a framework for understanding the functional effect of the disease-related mutations of hDNMT1.

\section{Results}

hDNMT1 $1_{351-1600}$ adopts an autoinhibitory conformation similar to that of $m D N M T 1_{291-1620}$ - The structure of hDNMT1 $1_{351-1600}$ reveals a closely-stacked three-layer architecture (Fig. 1A), with the RFTS and CXXC domains on the top, the MTase domain in the middle, and the two BAH domains situated at the bottom. In addition, it contains five zinc finger clusters, spreading over the RFTS domain, the CXXC domain, the BAH1 domain and the MTase domain, and an AdoHcy molecule in the active site (Fig. 1A). The Nterminal domains, except for the CXXC domain, make extensive contacts with the MTase domain, resulting in a compact fold (Fig. 1A). We have performed small X-ray scatting (SAXS) analysis to validate the observed structural fold (Fig. 1B). Our results revealed that the structure of hDNMT1 $1_{351-1600}$ fits well with the SAXS scatting curve, with a $\chi^{2}$ of 1.4 (Fig. 1B), thereby confirming that this structure is consistent with the conformation of hDNMT1 $1_{351-1600}$ in solution state.

The structure of hDNMT1 $1_{351-1600}$ reveals that its RFTS domain is anchored to the catalytic cleft of its MTase domain (the contacts are detailed below), potentially inhibiting the substrate binding (Fig. 1A). Such an autoinhibitory conformation is reminiscent of what was previously observed for $\mathrm{mDNMT1}_{291-1620}$ (Fig. 1C) (19). In fact, the structures of $h D N M T 1_{351-1600}$ and $m D N M T 1_{291-1620}$ superimpose well, with a root-mean-square deviation (r.m.s.d.) of $1.0 \AA$ over 989 aligned $\mathrm{C} \alpha$ atoms, consistent with the fact that these two orthologues share $\sim 83 \%$ sequence identity in this region (Fig. S1). The main conformational difference between the two structures arises from the N-terminal half of the RFTS domain, which is associated with relatively lower (72\%) sequence identity (Fig. S1). In particular, the $\alpha$-helix $\left(\alpha_{\text {RS: }}\right.$ : residues 495-519 in hDNMT1) that connects the two halves of the RFTS domain adopts a straight conformation in $\mathrm{hDNMT}_{351-1600}$, but is kinked in the structure of $\mathrm{mDNMT}_{291-1620}$ (Fig. 1C). As a result, the orientation of the first half of the RFTS domain relative to the rest of the protein is deviated by $19^{\circ}$ 
between $\mathrm{hDNMT} 1_{351-1600}$ and $\mathrm{mDNMT}_{291-1620}$ (Fig. 1C), despite the relatively conserved fold of this region. Such a conformational divergence between $\mathrm{hDNMT1}_{351-1600}$ and $\mathrm{mDNMT1}_{291-1620}$ is in attributed in part to their distinct molecular contacts within the RFTS domain (Fig. 1D,E). In the structure of hDNMT1 $_{351-1600}$, loop residues F469, E473, K474, A475 and I477 from the RFTS domain make close contacts with residues F499, Q502, Y507 and K510 from the N-terminal part of the $\alpha_{\text {RS }}$-helix (Fig. 1D). Whereas in the structure of $\mathrm{mDNMT} 1_{291-1620}$, the equivalent loop extends further toward the C-terminal end of the $\alpha_{\mathrm{RS}}$-helix, engaging in a distinct set of interactions that involve loop residues: L472, F475, D476, E479 and V481, and the $\alpha_{\text {RS }}$-helix residues: I512, K516, E520 and Q523 (Fig. 1E). In agreement with these conformational differences, fitting the structure of mDNMT1 (a region equivalent to hDNMT1 $1_{351-1600}$ ) to the hDNMT1 SAXS curve gives a worse fit than hDNMT1, with $\chi^{2}$ of 2.1 (Fig. S2).

The RFTS domain-mediated autoinhibition is supported by both direct domain-domain interactions and the CXXC-BAH1 domain linker - The previous structure of $\mathrm{mDNMT1}_{291-1620}$ has revealed that four hydrogen bonds, formed by residues E531, D532, D554 and L593 from the RFTS domain and K537, R1576, S1495 and T1505 from the MTase domain, respectively, mediate the association of the RFTS domain with the MTase domain (19). Inspection of the structure of hDNMT1 $1_{351-1600}$ not only confirms the existence of the equivalent interactions in hDNMT1 (Fig. 2A), but also reveals an hDNMT1-unique interaction that supports the contact between the RFTS and MTase domains: a hydrogen bond between residues D583 and $\mathrm{K} 1535$ of $\mathrm{hDNMT} 1_{351-1600}$. Such an interaction is absent in mDNMT1 due to the fact that the residue equivalent to D583 is replaced by an alanine (19). In addition, the improved resolution of the structure of hDNMT1 $1_{351-1600}$ has allowed us to identify a number of inter-domain contacts that have failed to be detected in the previous structure of $\mathrm{mDNMT}_{291-1600}$ (19). For instance, we have identified that two water-mediated hydrogen bonds, formed by the side chain of D548 with the backbone amide nitrogen of M1535 and the side chain hydroxyl group of Y1515, respectively, help mediate the association of the RFTS domain with the MTase domain (Fig. 2A). 
Analysis of the structure of $\mathrm{hDNMT} 1_{351-1600}$ reveals that the linker $\alpha$-helix between the CXXC and BAH1 domains also plays a role in associating the RFTS domain with the MTase domain. On one side, this helix interacts with the RFTS domain, through hydrogen bonds formed by the side chain of residue D700 and the side chains of residues R582 and K586, and by the side chain of residue E703 and the side chain of residue R582 (Fig. 2B). On the other side, this helix makes contacts with the MTase domain through hydrogen bonds formed by the side chain of residue D702 and the backbone and side chain of residue N1233, and a main chain hydrogen bond between residues D702 and M1235 (Fig. 2B). Together, these interactions further strengthen the association between the RFTS domain and the MTase domain.

Inhibition of the enzymatic activity of $h D N M T 1_{351-1600}$ by the $C X X C-B A H 1$ domain linker - To test the role of the CXXC-BAH1 domain linker in the RFTS domain-mediated autoinhibition of hDNMT1, we have mutated residue R582 to glutamate to disrupt its interaction with the helical linker, and analyzed the hDNMT1 $1_{351-1600}$-DNA interaction using Electrophoretic Mobility Shift Assay (EMSA). Our results showed that the R582E mutation indeed modestly increases the binding affinity of hDNMT1 ${ }_{351-1600}$ to a 26-mer hemimethylated CpG DNA (Fig. S3A, B). We then compared the enzymatic activities of wildtype and $\mathrm{R} 582 \mathrm{E} \mathrm{hDNMT1}_{351-1600}$ proteins using a mass spectrometry-based approach. The methylation reactions were carried out with a 12-mer hemimethylated DNA substrate containing one central CpG site (upper strand: 5'-GAGGC ${ }^{\mathrm{m}}$ CGCCTGC-3'; lower strand: 5'-GCAGG ${ }^{\mathrm{m}} \mathrm{CGGCCTC}^{\prime}$ ')' The activities of wild-type and R582E hDNMT1 $1_{351-1600}$ were measured using a previously published HPLC coupled with tandem mass spectrometry (LC-MS/MS) method (26,27). Quantitative analysis of the level of methylation was carried out based on the composite tandem mass spectrum of the $[\mathrm{M}-3 \mathrm{H}]^{3-}$ ions $(\mathrm{m} / \mathrm{z}$ 1218.9) of the two methylated strands (Fig. 3A, B). Along this line, collisional activation of deprotonated ions of short DNA gave rise to the cleavage of the $\mathrm{N}$-glycosidic bond and the 3' C-O bond within the same nucleoside to yield $\left[a_{n}-\right.$ base] and $w_{n}$ series ions (28). As displayed in Fig. 3A, some $\left[a_{n}-\right.$ base $]$ and $w_{n}$ ions for the two DNA strands share the same $m / z$ values (e.g. $\mathrm{w}_{5}, \mathrm{w}_{6}$ and $\mathrm{w}_{7}$ ions), whereas others have unique $\mathrm{m} / \mathrm{z}$ values. Considering that the upper strand is completely methylated, we estimated the level of 
methylation in the lower strand, expressed as \%methylation, based on the relative signal intensities observed for selected fragment ions for the two methylated DNA strands that exhibit unique $\mathrm{m} / \mathrm{z}$ values. In particular, we derived the \%methylation by dividing the peak area found in the selected-ion chromatogram (SIC) for monitoring the four transitions corresponding to the formations of the [ $\mathrm{a}_{3}$-base], $\mathrm{w}_{3}$, [a $\mathrm{a}_{4}$-base], and $\mathrm{w}_{4}$ ions, i.e. $\mathrm{m} / \mathrm{z} 1218.9 \rightarrow \mathrm{m} / \mathrm{z} 715.1,899.1,1028.2,1188.0$, for the lower strand by that found in the SIC for the corresponding transitions for the upper strand, i.e. $\mathrm{m} / \mathrm{z} 1218.9 \rightarrow \mathrm{m} / \mathrm{z} 739.0$, 939.0, 1068.2, 1228.1 (Fig. 3B). Despite the fact that the aforementioned peak area ratio may deviate to some extent from the \%methylation of the lower stand due to the differences in ionization and fragmentation efficiencies of the two DNA strands, this method permits a direct comparison of the relative levels of methylation induced by wild-type or mutant hDNMT1 proteins. As shown in Fig. 3C, at $2 \mathrm{hr}$ reaction time, we observed a $\sim 69 \%$ increase in the methylation activity of $\mathrm{hDNMT} 1_{351-1600}$ by the R582E mutation. The methylation activity of $\mathrm{hDNMT} 1_{351-1600}$ becomes even higher when we further removed a patch of residues (residues 694-701; the resulting mutant is denoted as $4694-701$ ) in the helical linker (Fig. 3C). These data confirm that the CXXC-BAH1 linker-mediated interactions are inhibitory to the enzymatic activity of DNMT1. Note that the methylation activity of the R582E mutant remains lower than that of an RFTS-free DNMT1 fragment, hDNMT1 ${ }_{646-1600}$ (Fig. S3C), indicating that the direct interactions between the RFTS and MTase domains are also important for the RFTS domain-mediated autoinhibtion (Fig. 2A) (20-22). Next, we performed SAXS analysis to examine whether these mutations affect the conformational states of hDNMT1 $1_{351-1600}$ in solution (Fig. S4A-D). We observe that the radius of gyration $(\mathrm{Rg})$ of $\mathrm{hDNMT} 1_{351-1600}$ increases from $40.5 \AA$ for wild-type protein to $48.0 \AA$ for the R582E mutant, and to $60.8 \AA$ for the $\Delta 694-701$ mutant, while its maximum dimension (Dmax) increases from 98 $\AA$ for wild-type protein to $163 \AA$ for the R582E mutant, and to $194 \AA$ for the $\Delta 694-701$ mutant (Fig. $\mathrm{S} 4 \mathrm{E})$. The increased $\mathrm{Rg}$ and Dmax values by the R582E and $\Delta 694-701$ mutations suggest these two mutations shift the conformational states of $\mathrm{hDNMT} 1_{351-1600}$ to a more open and elongated form. Together, 
these data support that the CXXC-BAH1 helical linker constitutes a key regulatory element in the RFTS domain-mediated autoinhibition of DNMT1.

Notably, the CXXC-BAH1 helical linker adopts an extended form in the structures of hDNMT1 $1_{646-1600}$ in complex with an unmethylated 19-mer CpG DNA (18) and free hDNMT1 $1_{601-1600}$ (PDB: 2SWR), suggesting that a helix-to-loop conformational transition was induced by the removal of the RFTS domain (Fig. S5). In both structures, this sequence is positioned along the catalytic cleft of hDNMT1 via its interaction with the MTase domain, thereby inhibiting the interaction between DNMT1 and DNA substrates (18). The inhibitory role of the CXXC-BAH1 linker in hDNMT1 $1_{646-1600}$ is further supported by our observation that the $\mathrm{hDNMT1} 1_{351-1600} \Delta 694-701$ mutant shows even higher methylation efficiency than hDNMT1 $1_{646-1600}$ (Fig. S3C). Taken together, these observations suggest that the CXXC-BAH1 domain linker inhibits the enzymatic activity of DNMT1 through a multi-layered mechanism.

The catalytic loops of AdoHcy-bound $h D N M T 1_{351-1600}$ and DDNMT1 $_{291-1620}$ assume different conformations - Previous studies on the DNA methyltransferase from Haemophilus haemolyticus (M.HhaI) or $\mathrm{mDNMT1}_{291-1620}$ have demonstrated that the catalytic site cysteine in the PCQ loop undergoes a conformational rearrangement in response to cofactor or DNA binding $(19,29,30)$. For instance, the catalytic residue $\mathrm{C} 1229$ of $\mathrm{mDNMT}_{291-1620}$ faces away from the DNA binding pocket in free state or AdoHcy-bound form; upon binding to AdoMet, it turns to approach the position where the flipped-out target cytosine is hosted (19). Based on this observation, it has been proposed that binding of the cofactor AdoMet provides a mechanism in switching the side chain conformation of the catalytic cysteine in mDNMT1 (19). Different from that of $\mathrm{mDNMT1}_{291-1620}$, the structure of $\mathrm{hDNMT1}_{351-1600}$ reveals that the active site cysteine, $\mathrm{C} 1226$, is poised to receive the target cytosine even in the presence of AdoHcy (Fig. 4A), suggesting that AdoMet-binding does not serve as a switch for the side chain conformation of C1226 in hDNMT1. Structural comparison of AdoHcy-bound hDNMT1 ${ }_{351-1600}$ and mDNMT1 $1_{291-1620}$ further indicates that such a conformational difference arises from the fact that residue C1226 in hDNMT1 experiences more steric repulsion from the phenyl group of the adjacent residue, 
F1229, than its counterpart in mDNMT1 (Fig. 4A). In fact, the conformation of the F1229 equivalent residue in mDNMT1 (F1232) is restrained by a hydrogen bond between the side chain of residue F1232 and the backbone of residue R1285; such a hydrogen bond is not formed in hDNMT1, since the residue equivalent to mDNMT1 R1285 is replaced by a lysine. These observations imply a different cofactor binding response in the catalytic site between hDNMT1 and mDNMT1. Interestingly, the conformation of the catalytic cysteine in $\mathrm{hDNMT} 1_{351-1600}$ is also different from that in the hDNMT1 $1_{646-1600}$-DNA complex (Fig. 4B). In the latter case, the CXXC-BAH1 domain linker occupies the DNA binding site, thereby pushing the side chain of residue C1226 away from the DNA binding pocket through steric hindrance.

Dynamic positioning of the CXXC domain between different functional states of hDNMT1 - The CXXC domain in the structure of mDNMT1 $1_{291-1620}$ was only partially determined (19), presumably due to the relatively low resolution and conformational dynamics. The improved resolution of the structure of hDNMT1 $1_{351-1600}$ in this study has permitted us to trace the entire CXXC domain (Fig. 1A and 4C). On this basis, we were able to compare the structure of the CXXC domain in free hDNMT1 (this study) and in its DNA-bound form (18), which reveals a surprisingly high conformational identity between the two forms of the CXXC domain (Fig. 4C). In particular, the CpG-recognition loop of the CXXC domain is well poised for DNA binding even in free state, suggestive of a preconfigured, functional conformation adopted by the CXXC domain. On the other hand, a large repositioning of the CXXC domain occurred between these two alternative states: The CXXC domain is spatially proximal to the MTase domain in free $\mathrm{hDNMT} 1_{351-1600}$, but shifts away by a distance of $\sim 30 \AA$ when a 19 -mer unmethylated CpG DNA is bound (Fig. 4C). As described above, such repositioning of the CXXC domain is coupled with a helix-toloop conformational transition for the linker sequence between the $\mathrm{CXXC}$ domain and the $\mathrm{BAH} 1$ domain, which then occupies the catalytic cleft to impose another layer of autoinhibition on DNMT1.

Structural analysis of the disease-related DNMT1 mutations - Recent evidence has revealed that mutations of DNMT1 are implicated in several neurological disorders (31-34), including hereditary sensory autonomic neurophathy with dementia and hearing loss (HSAN1E) and cerebella ataxia, deafness 
and narcolepsy (ADCA-DN). These mutations mainly fall into the RFTS domain of DNMT1 (Fig. 5A). For instance, mutations of D490E, P491Y, Y495C and Y495H were identified in a number of HSAN1E kindreds $(31,32)$; whereas mutations of A554V, C580R, G589A and V590F were implicated in a cohort of ADCA-DN patients $(33,34)$. Structural analysis of $\mathrm{hDNMT1}_{351-1600}$ suggests that some of these mutations may result in altered conformation and stability of DNMT1. For instance, the side chain hydroxyl group of residue Y495 forms a hydrogen bond with the main chain carbonyl oxygen of residue C420, which helps stabilize the orientation of $\alpha_{R S}$-helix in the RFTS domain (Fig. 5B). Mutation of Y495C or Y495H may disrupt such an interaction, leading to structural and dynamic change of the $\alpha_{\mathrm{RS}^{-}}$ helix. In addition, residues A554 and V590 both participate in formation of a hydrophobic cluster (Fig. 5C). Therefore, their respective mutations likely result in local structural perturbation of this region. Consistently, a previous study has shown that the Y495C mutation leads to reduced protein stability and enzymatic activity, and aberrant DNA methylation patterns (32). Further analysis of these disease-related mutations requires a detailed functional characterization of the RFTS domain of DNMT1.

\section{Discussion}

Recent structural and functional studies on DNMT1 have provided important insights into the allosteric regulation and substrate specificity of DNMT1 (18,20-22,35-37). However, the fact that hDNMT1 and mDNMT1 show slightly different enzymatic properties $(18,23-25)$ raises a question about whether structural analysis of mDNMT1 is sufficient to reveal the functional states of hDNMT1. This study presents the structure of an hDNMT1 construct that is comprised of all its structural domains, which therefore provides a framework for understanding of the structure-function relationship of hDNMT1, as well as the relationship between hDNMT1 mutations and human diseases.

The DNMT1 N-terminal domains are important in regulating the recruitment and enzymatic activity of DNMT1. The RFTS domain has been identified as a protein interaction module that mediates the recruitment of DNMT1 to replication foci $(14,16)$, and the interaction between DNMT1 and UHRF1 
$(20,21,38,39)$. However, the molecular basis underlying these regulations remains unknown. Our structural study reveals detailed molecular interactions between the RFTS domain and the MTase domain, which explains the autoinhibitory role of the RFTS domain in the enzymatic activity of hDNMT1. In addition, we observed a conformational difference of the RFTS domain between $\mathrm{hDNMT1}_{351-1600}$ and mDNMT1 $1_{291-1620,}$ which, despite the possible contribution of different crystallization conditions, implies subtle structural diversity between the two orthologues.

Structural analysis and biochemical assays of $\mathrm{hDNMT} 1_{351-1600}$ reveal that the helical linker between the CXXC domain and the BAH1 domain constitutes an important regulatory element in the RFTS domain-mediated autoinhibition. It interacts with both the RFTS domain and the MTase domain, thereby strengthening the association of the RFTS domain with the MTase domain. Notably, this CXXC-BAH1 helical linker undergoes a helix-to-loop conformational transition when DNMT1 binds to unmethylated CpG DNA (18), positioned along the catalytic cleft of DNMT1 to inhibit the interaction between DNMT1 and DNA substrates (18). Together, these observations suggest that the CXXC-BAH1 domain linker, namely autoinhibitory linker, inhibits the enzymatic activity of DNMT1 through a multi-layered mechanism (Fig. 6). Such inhibition of DNMT1 will be conceivably released when DNMT1 binds to hemimethylated CpG DNA substrates, accompanied by a large conformational rearrangement of the RFTS and CXXC domains (Fig. 6), although the conformation of the autoinhibitory linker under this state is currently unknown.

In comparison with the structure of $\mathrm{mDNMT}_{291-1620}$, this study reveals a number of distinct structural features for hDNMT1, including its catalytic site. In mDNMT1, the active site cysteine faces away from the DNA binding pocket in the presence of AdoHcy, but turns to approach the position where the target cytosine is hosted in the presence of AdoMet. Based on these observations, it has been proposed that the active site cysteine of mDNMT1 switches from an inactive conformation to an active conformation upon binding to AdoMet, but returns back to an inactive conformation after the methylation reaction occurs (19). By contrast, the side chain of the catalytic cysteine of hDNMT1 is poised to receive the target 
cytosine even in the presence of AdoHcy. Therefore, the AdoMet/AdoHcy cofactor binding does not appear to provide a switching mechanism for the active site of hDNMT1. Such divergence in functional regulation of hDNMT1 and mDNMT1 likely contributes to their slightly different enzymatic behaviors $(23-25)$.

\section{Materials and Methods}

\section{Protein expression and purification}

The gene encoding hDNMT1 $1_{351-1600}$ was amplified by PCR and inserted into a modified pRSFDuet-1 vector, where it was preceded by an N-terminal His6-tag and a SUMO-tag. The sequence of hDNMT1 $1_{351-}$ 1600 was confirmed by DNA sequencing analysis. The hDNMT1 $1_{351-1600}$ construct used for crystallization contains an additional five-residue patch (LTRVW) introduced to the very C-terminus, due to cloning artifacts. The R582E and $\Delta 694-701$ mutants were obtained through site-directed mutagenesis. The plasmids of $\mathrm{hDNMT1}_{351-1600}$ constructs were transformed into BL21 (DE3) RIL cell strain for overexpression. The bacterial cells were grown at $37{ }^{\circ} \mathrm{C}$ and induced by $0.4 \mathrm{mM}$ isopropyl $\beta$-d-1thiogalactopyranoside (IPTG) when the cells density reached an optical density $\left(\mathrm{OD}_{600}\right)$ of 0.8 . After induction, the cells continued to grow at $16{ }^{\circ} \mathrm{C}$ for $16 \mathrm{hrs}$. The cells were then harvested, and lysed in a buffer containing $50 \mathrm{mM}$ Tris- $\mathrm{HCl}(\mathrm{pH} 8.0), 25 \mathrm{mM}$ imidazole, $1 \mathrm{M} \mathrm{NaCl}, 0.5 \mathrm{mM}$ DTT and $1 \mathrm{mM}$ PMSF. The His-SUMO-DNMT1 fusion protein was first purified by a Ni-NTA column, followed by removal of the His6-SUMO tag by Ubiquitin-like protease 1 (ULP1) cleavage. The protein sample for hDNMT1 $1_{351-1600}$ was further purified through ion exchange (Hitrap Q, GE Health Sciences) and sizeexclusion (Superdex 200 16/60, GE Health Sciences) chromatography methods. The final protein sample, with $>95 \%$ purity, was concentrated to $\sim 15 \mathrm{mg} / \mathrm{ml}$ and stored in a buffer containing $25 \mathrm{mM}$ Tris- $\mathrm{HCl}$ (pH 7.5), $100 \mathrm{mM} \mathrm{NaCl}$ and $5 \mathrm{mM}$ DTT at $-80{ }^{\circ} \mathrm{C}$.

\section{Crystallization and structure determination}


For crystallization, hDNMT1 $1_{351-1600}$ was first mixed with AdoHcy in a molar ratio of 1:1.5. The complex was then crystallized using sitting-drop vapor diffusion method by mixing $1.5 \mu 1$ of $\mathrm{hDNMT}_{351 \text { - }}$ 1600 sample and $1.5 \mu \mathrm{l}$ of reservoir solution containing $100 \mathrm{mM}$ MES, pH6.0, $50 \mathrm{mM} \mathrm{CaCl}$, and $45 \%$ PEG200 at $16^{\circ} \mathrm{C}$. Crystals that grew into full size in three days were equilibrated in reservoir solution before flash-frozen in liquid nitrogen.

The X-ray diffraction data set for $\mathrm{hDNMT}_{351-1600}$ were collected on the BL 5.0.1 beamline at the Advanced Light Source (ALS), Lawrence Berkeley National Laboratory. The diffraction data were indexed, integrated and scaled using the HKL 2000 program. The structure of hDNMT1 $1_{351-1600}$ was solved using the molecular replacement method in PHASER (40) using the structure of mDNMT1 $1_{291-1620}$ (PDB 3AV4) as a search model. The resulting electron density revealed that there are two molecules of hDNMT1 $1_{351-1600}$ in each asymmetric unit (ASU). Further modeling of the hDNMT1 $1_{351-1600}$ was carried out using COOT (41), and then subject to refinement using the PHENIX software package (42). The same Rfree test set was used throughout the refinement. The B-factors were refined with individual B values. The statistics for data collection and structural refinement of hDNMT1 $1_{351-1600}$ is summarized in Table 1.

\section{Small Angle X-ray Scatting experiment (SAXS)}

To measure the X-ray scatting curve, 2-6 mg/ml hDNMT1 $1_{351-1600}$ wild-type, R582E or $\Delta 694-701$ protein samples were dissolved in a buffer containing $20 \mathrm{mM}$ Tris- $\mathrm{HCl}, \mathrm{pH} 7.5,250 \mathrm{mM} \mathrm{NaCl}$ and $5 \mathrm{mM}$ DTT. The SAXS data were collected at the SYBIL beamline at ALS, Lawrence Berkeley National Laboratory, and processed using the ScÅtter software (https://bl1231.als.lbl.gov/scatter/). The scattering curves calculated from the crystal structures were fit with the experimental curve using the fast opensource X-ray scatting (FoXS) method (43) through the FOXS server (http://modbase.compbio.ucsf.edu/foxs/).

\section{Enzymatic methylation kinetics assay}


The DNMT1 cytosine-5 methylation assays were carried out in triplicates, using protocols described previously (26). 30 pmol of 12-mer DNA duplex (upper strand: 5'-GAGGC ${ }^{\mathrm{m}}$ CGCCTGC-3'; lower strand: 5'-GCAGGCGGCCTC-3') was incubated with 15 nmol of $S$-adenosyl-L-methionine (New England Biolabs, Ipswich, WA) and 3 pmol of wild-type hDNMT1 (or its R582E mutant) at $37{ }^{\circ} \mathrm{C}$ in a reaction buffer (50 mM Tris-HCl, $1 \mathrm{mM}$ DTT, 5\% glycerol, $100 \mu \mathrm{g} / \mathrm{mL}$ BSA, $\mathrm{pH}$ 7.5). After incubation for 0.5, 1.0, and $2.0 \mathrm{hr}$, the enzyme was inactivated by heating at $65^{\circ} \mathrm{C}$ for $20 \mathrm{~min}$ and removed by chloroform extraction, and the aqueous layer was subjected to LC-MS/MS analysis.

\section{LC-MS/MS Analysis and Data Processing}

The LC-MS/MS experiments were conducted on an LTQ linear ion-trap mass spectrometer (Thermo Fisher Scientific, San Jose, CA) equipped with an Agilent 1200 capillary HPLC (Agilent Technologies, Santa Clara, CA). A $0.5 \times 250 \mathrm{~mm}$ Zorbax SB-C18 column $(5 \mu \mathrm{m}$ in particle size, Agilent Technologies, Santa Clara, CA) was employed for the separation at a flow rate of $8.0 \mu \mathrm{L} / \mathrm{min}$ and with a gradient of 20 min of 5\% methanol followed by $20 \mathrm{~min}$ of $65 \%$ methanol in $400 \mathrm{mM} \mathrm{1,1,1,3,3,3-hexafluoro-2-propanol}$ $(\mathrm{pH} 7.0$, adjusted with triethylamine). The spray, capillary and tube lens voltages were $4.5 \mathrm{kV},-31 \mathrm{~V}$ and $-95 \mathrm{~V}$, respectively. The temperature for the ion transport tube was maintained at $300^{\circ} \mathrm{C}$.

\section{EMSA (electrophoretic mobility shift assay)}

A 26-bp hemimethylated DNA duplex (upper strand: 5'ACACCAAGCCTGmCGGAGGCTCACGGA -3', mC = 5-methyl-2'-deoxycytidine; lower strand: 5' TCCGTGAGCCTCCGCAGGCTTGGTGT -3') was used to characterize DNA binding of the wild-type $\mathrm{hDNMT}_{351-1600}$ and its R582E mutant. Binding reactions contained $0.1 \mu \mathrm{M}$ dsDNA and various concentrations of protein, dissolved in $10 \mu \mathrm{l}$ of binding buffer $(20 \mathrm{mM}$ Tris- $\mathrm{HCl}, \mathrm{pH} 7.5,50 \mathrm{mM} \mathrm{NaCl}$, $1 \mathrm{mM}$ DTT). Binding reaction mixtures were electrophoresed in 6\% DNA retardation gel (Invitrogen) in $0.25 \mathrm{X}$ TBE ( $89 \mathrm{mM}$ Tris-borate, $\mathrm{pH} 8.4$ ) buffer at $4{ }^{\circ} \mathrm{C}$ under $100 \mathrm{~V}$ for $50 \mathrm{~min}$. DNA was visualized by SYBR green staining and quantitated with imageJ software (44). 


\section{PDB accession numbers}

The atomic coordinates and structural factors have been deposited into the PDB under the accession code 4WXX.

\section{Acknowledgements}

This work was supported by UCR Regent Fellowship and Basil O’Connor Starter Scholar Research Award, March of Dimes Foundation to J.S., and was provided in part by National Institutes of Health grant R01 CA101864 to Y.W. The Berkeley Center for Structural Biology is supported in part by the National Institutes of Health, National Institute of General Medical Sciences, and the Howard Hughes Medical Institute. The Advanced Light Source is supported by the Director, Office of Science, Office of Basic Energy Sciences, of the U.S. Department of Energy under Contract No. DE-AC02-05CH11231.

\section{References:}

1. Bourc'his, D., and Bestor, T. H. (2004) Meiotic catastrophe and retrotransposon reactivation in male germ cells lacking Dnmt3L. Nature 431, 96-99

2. Holliday, R., and Pugh, J. E. (1975) DNA modification mechanisms and gene activity during development. Science 187, 226-232

3. Walsh, C. P., Chaillet, J. R., and Bestor, T. H. (1998) Transcription of IAP endogenous retroviruses is constrained by cytosine methylation. Nat Genet 20, 116-117

4. Li, E., Beard, C., and Jaenisch, R. (1993) Role for DNA methylation in genomic imprinting. Nature 366, 362-365

5. Panning, B., and Jaenisch, R. (1998) RNA and the epigenetic regulation of X chromosome inactivation. Cell 93, 305-308

6. Ehrlich, M., Gama-Sosa, M. A., Huang, L. H., Midgett, R. M., Kuo, K. C., McCune, R. A., and Gehrke, C. (1982) Amount and distribution of 5-methylcytosine in human DNA from different types of tissues of cells. Nucleic Acids Res 10, 2709-2721

7. Goll, M. G., and Bestor, T. H. (2005) Eukaryotic cytosine methyltransferases. Annu Rev Biochem 74, 481-514

8. Jones, P. A., and Liang, G. (2009) Rethinking how DNA methylation patterns are maintained. Nat Rev Genet 10, 805-811

9. Reik, W., Dean, W., and Walter, J. (2001) Epigenetic reprogramming in mammalian development. Science 293, 1089-1093

10. Riggs, A. D., and Xiong, Z. (2004) Methylation and epigenetic fidelity. Proc Natl Acad Sci U S A 101, 4-5

11. Gruenbaum, Y., Cedar, H., and Razin, A. (1982) Substrate and sequence specificity of a eukaryotic DNA methylase. Nature 295, 620-622 
12. Yoder, J. A., Soman, N. S., Verdine, G. L., and Bestor, T. H. (1997) DNA (cytosine-5)methyltransferases in mouse cells and tissues. Studies with a mechanism-based probe. $J \mathrm{Mol}$ Biol 270, 385-395

13. Easwaran, H. P., Schermelleh, L., Leonhardt, H., and Cardoso, M. C. (2004) Replicationindependent chromatin loading of Dnmt1 during $G 2$ and $M$ phases. EMBO Rep 5, 1181-1186

14. Leonhardt, H., Page, A. W., Weier, H. U., and Bestor, T. H. (1992) A targeting sequence directs DNA methyltransferase to sites of DNA replication in mammalian nuclei. Cell 71, 865-873

15. Chuang, L. S., Ian, H. I., Koh, T. W., Ng, H. H., Xu, G., and Li, B. F. (1997) Human DNA-(cytosine-5) methyltransferase-PCNA complex as a target for p21WAF1. Science 277, 1996-2000

16. Nishiyama, A., Yamaguchi, L., Sharif, J., Johmura, Y., Kawamura, T., Nakanishi, K., Shimamura, S., Arita, K., Kodama, T., Ishikawa, F., Koseki, H., and Nakanishi, M. (2013) Uhrf1-dependent H3K23 ubiquitylation couples maintenance DNA methylation and replication. Nature 502, 249-253

17. Cheng, X., and Blumenthal, R. M. (2008) Mammalian DNA methyltransferases: a structural perspective. Structure 16, 341-350

18. Song, J., Rechkoblit, O., Bestor, T. H., and Patel, D. J. (2011) Structure of DNMT1-DNA complex reveals a role for autoinhibition in maintenance DNA methylation. Science 331, 1036-1040

19. Takeshita, K., Suetake, I., Yamashita, E., Suga, M., Narita, H., Nakagawa, A., and Tajima, S. (2011) Structural insight into maintenance methylation by mouse DNA methyltransferase 1 (Dnmt1). Proc Natl Acad Sci U S A 108, 9055-9059

20. Bashtrykov, P., Rajavelu, A., Hackner, B., Ragozin, S., Carell, T., and Jeltsch, A. (2014) Targeted mutagenesis results in an activation of DNA methyltransferase 1 and confirms an autoinhibitory role of its RFTS domain. Chembiochem : a European journal of chemical biology 15, 743-748

21. Berkyurek, A. C., Suetake, I., Arita, K., Takeshita, K., Nakagawa, A., Shirakawa, M., and Tajima, S. (2014) The DNA methyltransferase Dnmt1 directly interacts with the SET and RING fingerassociated (SRA) domain of the multifunctional protein Uhrf1 to facilitate accession of the catalytic center to hemi-methylated DNA. J Biol Chem 289, 379-386

22. Syeda, F., Fagan, R. L., Wean, M., Avvakumov, G. V., Walker, J. R., Xue, S., Dhe-Paganon, S., and Brenner, C. (2011) The replication focus targeting sequence (RFTS) domain is a DNA-competitive inhibitor of Dnmt1. J Biol Chem 286, 15344-15351

23. Flynn, J., Glickman, J. F., and Reich, N. O. (1996) Murine DNA cytosine-C5 methyltransferase: pre-steady- and steady-state kinetic analysis with regulatory DNA sequences. Biochemistry 35, 7308-7315

24. Pradhan, S., Bacolla, A., Wells, R. D., and Roberts, R. J. (1999) Recombinant human DNA (cytosine-5) methyltransferase. I. Expression, purification, and comparison of de novo and maintenance methylation. J Biol Chem 274, 33002-33010

25. Pradhan, S., Talbot, D., Sha, M., Benner, J., Hornstra, L., Li, E., Jaenisch, R., and Roberts, R. J. (1997) Baculovirus-mediated expression and characterization of the full-length murine DNA methyltransferase. Nucleic Acids Res 25, 4666-4673

26. Ji, D., Lin, K., Song, J., and Wang, Y. (2014) Effects of Tet-induced oxidation products of 5methylcytosine on Dnmt1- and DNMT3a-mediated cytosine methylation. Molecular bioSystems 10, 1749-1752

27. Wang, H., and Wang, Y. (2009) 6-Thioguanine perturbs cytosine methylation at the CpG dinucleotide site by DNA methyltransferases in vitro and acts as a DNA demethylating agent in vivo. Biochemistry 48, 2290-2299

28. McLuckey, S. A., Van Berkel, G. J., and Glish, G. L. (1992) Tandem mass spectrometry of small, multiply charged oligonucleotides. Journal of the American Society for Mass Spectrometry 3, 6070 
29. Cheng, X., Kumar, S., Posfai, J., Pflugrath, J. W., and Roberts, R. J. (1993) Crystal structure of the Hhal DNA methyltransferase complexed with S-adenosyl-L-methionine. Cell 74, 299-307

30. Klimasauskas, S., Kumar, S., Roberts, R. J., and Cheng, X. (1994) Hhal methyltransferase flips its target base out of the DNA helix. Cell 76, 357-369

31. Klein, C. J., Bird, T., Ertekin-Taner, N., Lincoln, S., Hjorth, R., Wu, Y., Kwok, J., Mer, G., Dyck, P. J., and Nicholson, G. A. (2013) DNMT1 mutation hot spot causes varied phenotypes of HSAN1 with dementia and hearing loss. Neurology 80, 824-828

32. Klein, C. J., Botuyan, M. V., Wu, Y., Ward, C. J., Nicholson, G. A., Hammans, S., Hojo, K., Yamanishi, H., Karpf, A. R., Wallace, D. C., Simon, M., Lander, C., Boardman, L. A., Cunningham, J. M., Smith, G. E., Litchy, W. J., Boes, B., Atkinson, E. J., Middha, S., PJ, B. D., Parisi, J. E., Mer, G., Smith, D. I., and Dyck, P. J. (2011) Mutations in DNMT1 cause hereditary sensory neuropathy with dementia and hearing loss. Nat Genet 43, 595-600

33. Pedroso, J. L., Povoas Barsottini, O. G., Lin, L., Melberg, A., Oliveira, A. S., and Mignot, E. (2013) A novel de novo exon 21 DNMT1 mutation causes cerebellar ataxia, deafness, and narcolepsy in a Brazilian patient. Sleep 36, 1257-1259, 1259A

34. Winkelmann, J., Lin, L., Schormair, B., Kornum, B. R., Faraco, J., Plazzi, G., Melberg, A., Cornelio, F., Urban, A. E., Pizza, F., Poli, F., Grubert, F., Wieland, T., Graf, E., Hallmayer, J., Strom, T. M., and Mignot, E. (2012) Mutations in DNMT1 cause autosomal dominant cerebellar ataxia, deafness and narcolepsy. Human molecular genetics 21, 2205-2210

35. Song, J., Teplova, M., Ishibe-Murakami, S., and Patel, D. J. (2012) Structure-based mechanistic insights into DNMT1-mediated maintenance DNA methylation. Science 335, 709-712

36. Svedruzic, Z. M., and Reich, N. O. (2005) DNA cytosine C5 methyltransferase Dnmt1: catalysisdependent release of allosteric inhibition. Biochemistry 44, 9472-9485

37. Svedruzic, Z. M., and Reich, N. O. (2005) Mechanism of allosteric regulation of Dnmt1's processivity. Biochemistry 44, 14977-14988

38. Achour, M., Jacq, X., Ronde, P., Alhosin, M., Charlot, C., Chataigneau, T., Jeanblanc, M., Macaluso, M., Giordano, A., Hughes, A. D., Schini-Kerth, V. B., and Bronner, C. (2008) The interaction of the SRA domain of ICBP90 with a novel domain of DNMT1 is involved in the regulation of VEGF gene expression. Oncogene 27, 2187-2197

39. Felle, M., Joppien, S., Nemeth, A., Diermeier, S., Thalhammer, V., Dobner, T., Kremmer, E., Kappler, R., and Langst, G. (2011) The USP7/Dnmt1 complex stimulates the DNA methylation activity of Dnmt1 and regulates the stability of UHRF1. Nucleic Acids Res 39, 8355-8365

40. McCoy, A. J., Grosse-Kunstleve, R. W., Adams, P. D., Winn, M. D., Storoni, L. C., and Read, R. J. (2007) Phaser crystallographic software. J App/ Crystallogr 40, 658-674

41. Emsley, P., and Cowtan, K. (2004) Coot: model-building tools for molecular graphics. Acta Crystallogr D Biol Crystallogr 60, 2126-2132

42. Adams, P. D., Grosse-Kunstleve, R. W., Hung, L. W., loerger, T. R., McCoy, A. J., Moriarty, N. W., Read, R. J., Sacchettini, J. C., Sauter, N. K., and Terwilliger, T. C. (2002) PHENIX: building new software for automated crystallographic structure determination. Acta Crystallogr D Biol Crystallogr 58, 1948-1954

43. Schneidman-Duhovny, D., Hammel, M., Tainer, J. A., and Sali, A. (2013) Accurate SAXS profile computation and its assessment by contrast variation experiments. Biophysical journal 105, 962974

44. Schneider, C. A., Rasband, W. S., and Eliceiri, K. W. (2012) NIH Image to ImageJ: 25 years of image analysis. Nature methods 9, 671-675 


\section{Figure Legends}

Figure 1. Structural overview of hDNMT1 ${ }_{351-1600}$ bound to AdoHcy. (A) Ribbon representation of hDNMT1 $1_{351-1600}$ in complex with AdoHcy, with the RFTS, CXXC, BAH1, BAH2 and MTase domains colored in green, red, pink, orange, and light blue, respectively. The domain linkers and zinc ions are colored in silver and purple, respectively. The bound AdoHcy is in a space filling representation. (B) Measured scattering curve of free hDNMT1 ${ }_{351-1600}$ (black) is compared with the scattering curve calculated from the crystal structure (red). (C) Superposition of $\mathrm{hDNMT} 1_{351-1600}$ (light blue) and mDNMT1 $1_{291-1620}$ (magenta), with the N-terminal half of the RFTS domains highlighted in an expanded view. (D-E) Interactions between a loop from the RFTS domain and the $\alpha_{\mathrm{RS}}$-helix in $\mathrm{hDNMT1}_{351-1600}$ (D) and $\mathrm{mDNMT} 1_{291-1620}(\mathbf{E})$, respectively.

Figure 2. Structural details of hDNMT1 ${ }_{351-1600}$ highlighting the RFTS domain-mediated autoinhibition. (A) Hydrogen bonding Interactions between the RFTS domain (green) and the MTase domain (light blue) in $\mathrm{hDNMT} 1_{351-1600}$. The hydrogen bonds are shown in dashed line. The water molecules are shown in sphere. (B) Hydrogen bonding interactions of the CXXC-BAH1 helical linker (grey) with the MTase and RFTS domains.

Figure 3. Enzymatic activities of wild type and mutant hDNMT1 $1_{351-1600}$. (A) LC-MS/MS characterization of upper DNA strand (5'-GAGGCmCGCCTGC-3', blue) and methylated lower DNA strand (5'-GCAGGmCGGCCTC-3', red) in 12mer-DNA duplex after reaction with hDNMT1 ${ }_{351-1600}$. The product-ion spectrum (MS/MS) of the $[\mathrm{M}-3 \mathrm{H}]^{3-}$ ion $(\mathrm{m} / \mathrm{z} 1218.9)$ is shown. (B) Quantification of the percentage of methylation by comparing the peak areas found in the selected-ion chromatograms (SICs) for monitoring the indicated transitions of upper DNA strand and newly methylated lower DNA strand. (C) Enzymatic kinetics of wild-type hDNMT1 and its R582E and $\Delta 694-701$ mutants.

Figure 4. The catalytic cysteine in hDNMT1 $_{351-1600}$ is poised to receive the target cytosine. (A) Superposition of the catalytic loop between hDNMT1 $1_{351-1600}$ (light blue) and $\mathrm{mDNMT1}_{291-1620}$ (magenta). The distance between the backbone carbonyl oxygen of F1229 and K1285 in hDNMT1 $151-1600$ is $4.6 \AA$, whereas the equivalent groups in mDNMT1 $1_{291-1620}$ form a hydrogen bond, with a distance of $3.0 \AA$. (B) Superposition of the catalytic loop between hDNMT1 $1_{351-1600}$ (light blue) and $\mathrm{hDNMT1}_{646-1600}-\mathrm{DNA}$ complex (wheat, PDB 3PTA). (C) The DNMT1 CXXC domain undergoes a large repositioning from the free state (light blue) to the DNA bound form (wheat). The CXXC domain in free hDNMT1 $1_{351-1600}$ and in complex with $\mathrm{CpG}$ DNA is superimposed in a rectangle.

Figure 5. Molecular interactions involving the disease-related mutation sites of hDNMT1. (A) Surface and ribbon representation of $\mathrm{hDNMT1} 1_{351-1600}$, with areas of the reported mutation sites in neurological disorders colored in red. (B) Hydrogen bond between the side chain of Y495 and the backbone carbonyl oxygen of C420 is shown in dashed line. (C) Residue A554 and V590 are involved in forming a hydrophobic cluster within the RFTS domain.

Figure 6. The CXXC-BAH1 linker plays a role in the autoinhibitory mechanisms of DNMT1. The CXXC-BAH1 helical linker (a.k.a. autoinhibitory linker) is colored green. The conformational transitions of hDNMT1 upon binding to different DNA substrates are proposed. 


\section{Table 1.Crystallographic statistics for hDNMT1 $1_{351-1600}$.}

\begin{tabular}{|c|c|}
\hline \multicolumn{2}{|l|}{ Data collection } \\
\hline Wavelength $(\AA)$ & 0.9774 \\
\hline Space group & $\mathrm{P}_{3}$ \\
\hline \multicolumn{2}{|l|}{ Cell dimensions } \\
\hline$a, b, c(\AA)$ & $138.6,138.6,221.5$ \\
\hline$\alpha, \beta, \gamma\left({ }^{\circ}\right)$ & $90,104.4,90$ \\
\hline Resolution $(\AA)$ & $50-2.62(2.71-2.62)^{\mathrm{a}}$ \\
\hline$R_{\text {merge }} \mathrm{b}^{\prime}$ & $11.8(75.5)$ \\
\hline$I / \sigma I$ & $12.8(2.1)$ \\
\hline Completeness (\%) & $98.6(99.0)$ \\
\hline Redundancy & $5.2(4.9)$ \\
\hline No. of unique reflections & $122,755(12,321)$ \\
\hline $\begin{array}{l}\text { Complex molecules/ } \\
\text { asymmetric unit }\end{array}$ & 2 \\
\hline \multicolumn{2}{|l|}{ Refinement } \\
\hline Resolution $(\AA)$ & $48.22-2.62$ \\
\hline No. reflections & 122,708 \\
\hline$R_{\text {work } /} R_{\text {free }}(\%)^{\mathrm{c}}$ & $19.3 / 24.0$ \\
\hline \multicolumn{2}{|l|}{ No. atoms } \\
\hline Protein & 18336 \\
\hline Ligand & 52 \\
\hline Zinc & 10 \\
\hline Water & 637 \\
\hline \multicolumn{2}{|l|}{ B-factors } \\
\hline Protein & 51.2 \\
\hline Ligand & 35.9 \\
\hline Zinc & 59.0 \\
\hline Water & 44.8 \\
\hline \multicolumn{2}{|l|}{ R.m.s deviations } \\
\hline Bond lengths $(\AA)$ & 0.009 \\
\hline Bond angles $\left({ }^{\circ}\right)$ & 1.400 \\
\hline \multicolumn{2}{|l|}{ Ramachandran plot } \\
\hline Favoured regions $(\%)$ & 91.7 \\
\hline Allowed regions (\%) & 6.7 \\
\hline Outliers $(\%)$ & 1.6 \\
\hline
\end{tabular}

${ }^{a}$. Highest resolution shell is shown in parenthesis.

b. $R_{\text {merge }}=\Sigma_{\text {hkl }} \Sigma_{\mathrm{i} \mid}\left|I_{i}(h k l)-<I(h k l)>\right| \Sigma_{\mathrm{hkl}} \Sigma_{\mathrm{i}} I_{i}(h k l)$, where $I_{i}(h k l)$ is the intensity of the $i^{\text {th }}$ observation of reflection $h k l$, and $\langle I(h k l)\rangle$ is the average over all observations of reflection $h k l$.

c. $R_{\text {factor }}=\Sigma_{h k l} \square \mathrm{F}_{\mathrm{o}}|-| \mathrm{Fc} \square / \Sigma_{h k l}\left|\mathrm{~F}_{\mathrm{o}}\right|$ for all data excluding the $5 \%$ that comprised the $R_{\text {free }}$ used for cross-validation. 
A

A
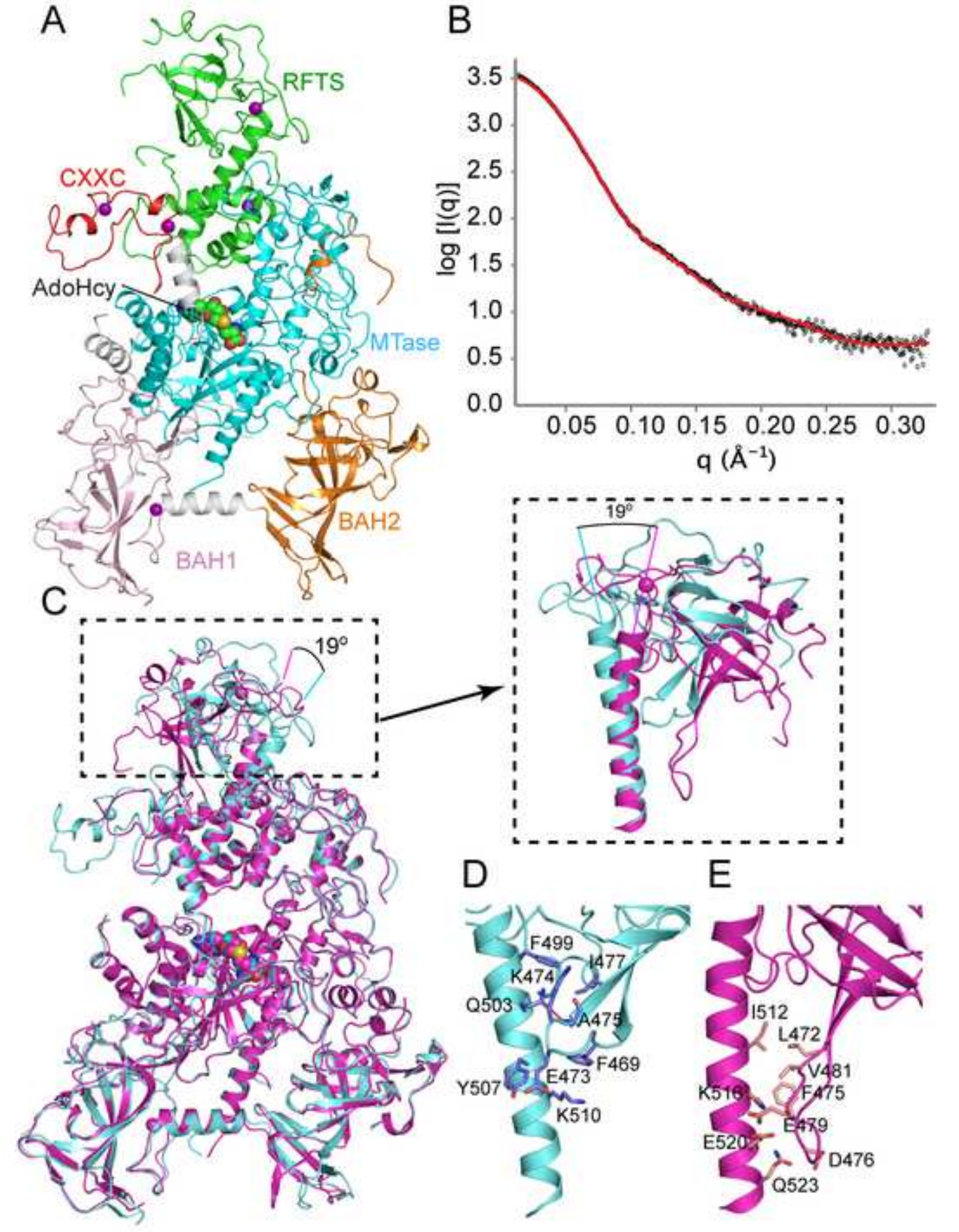


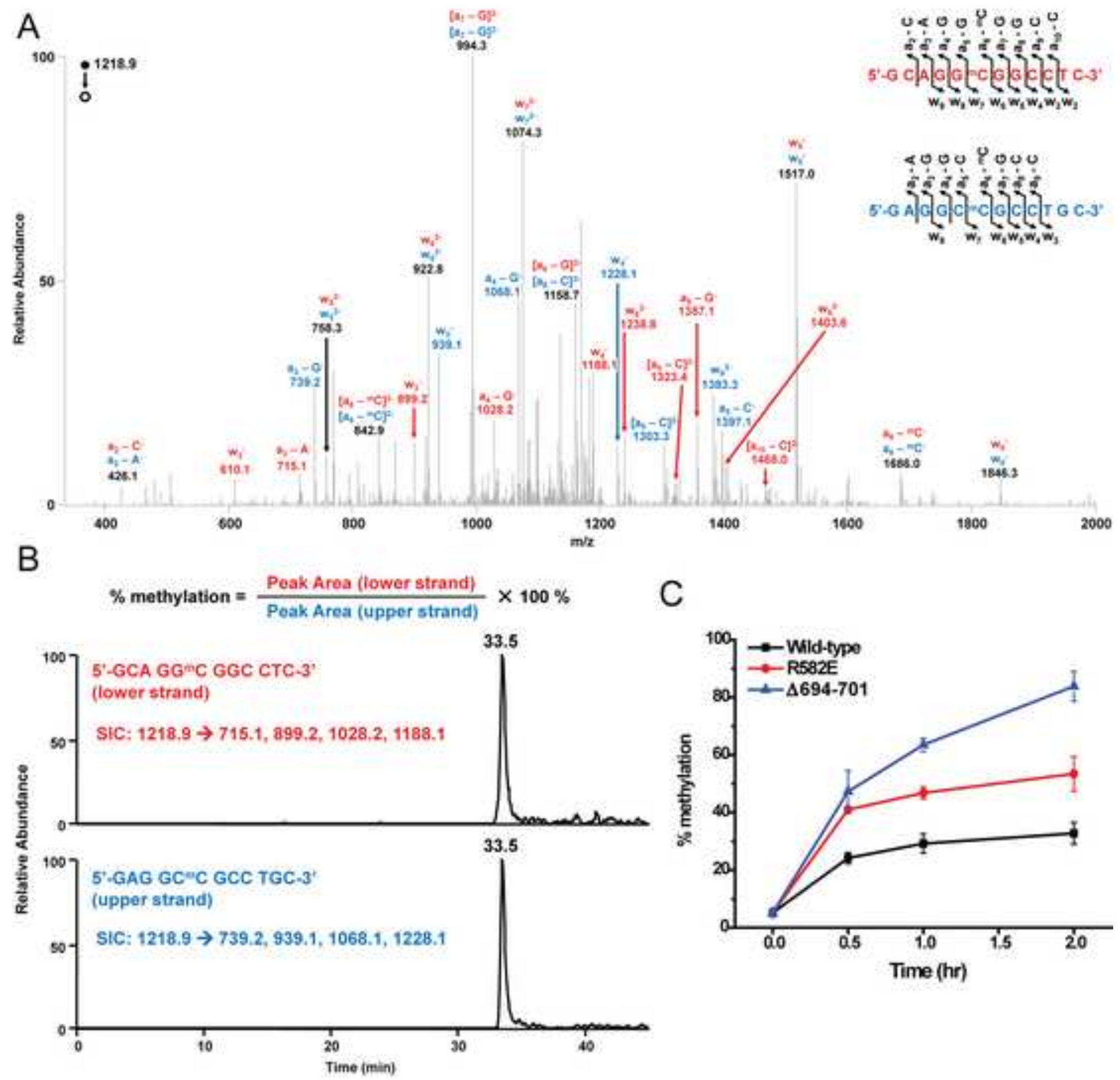



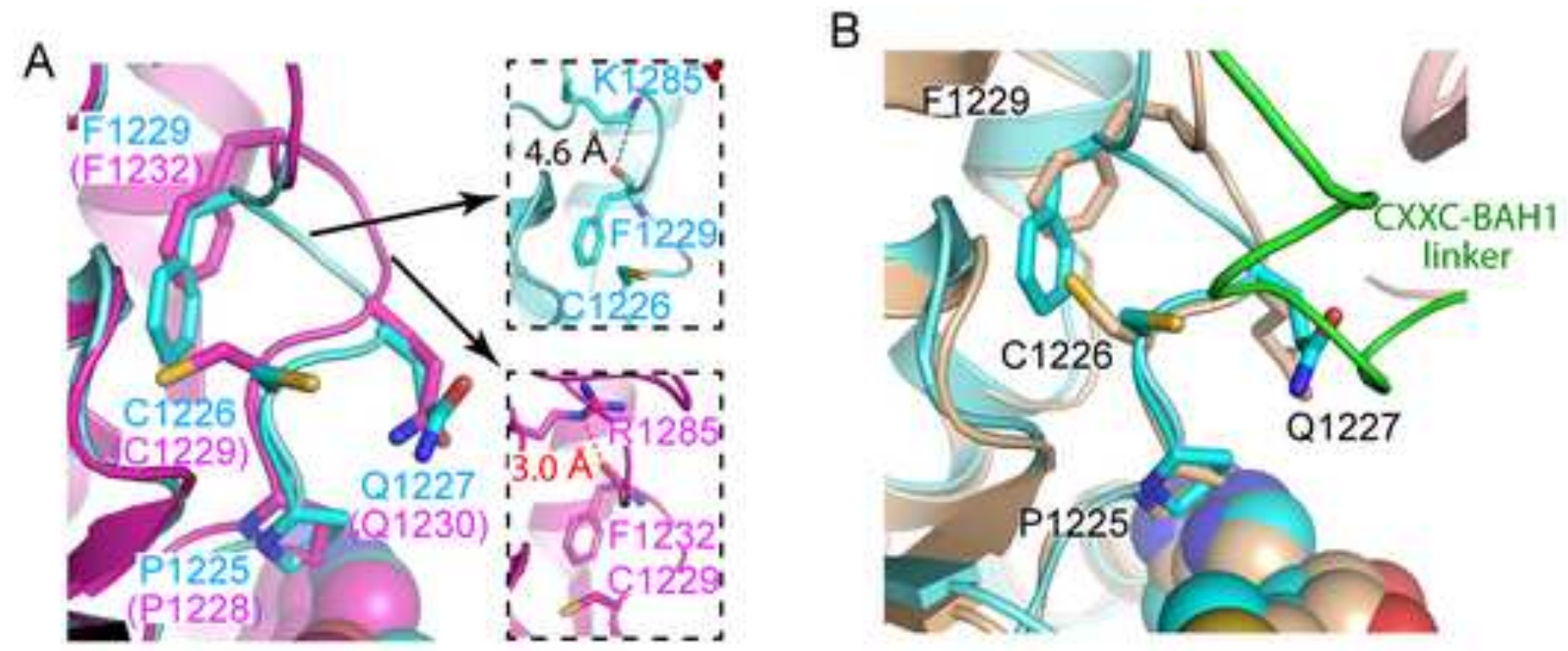

C
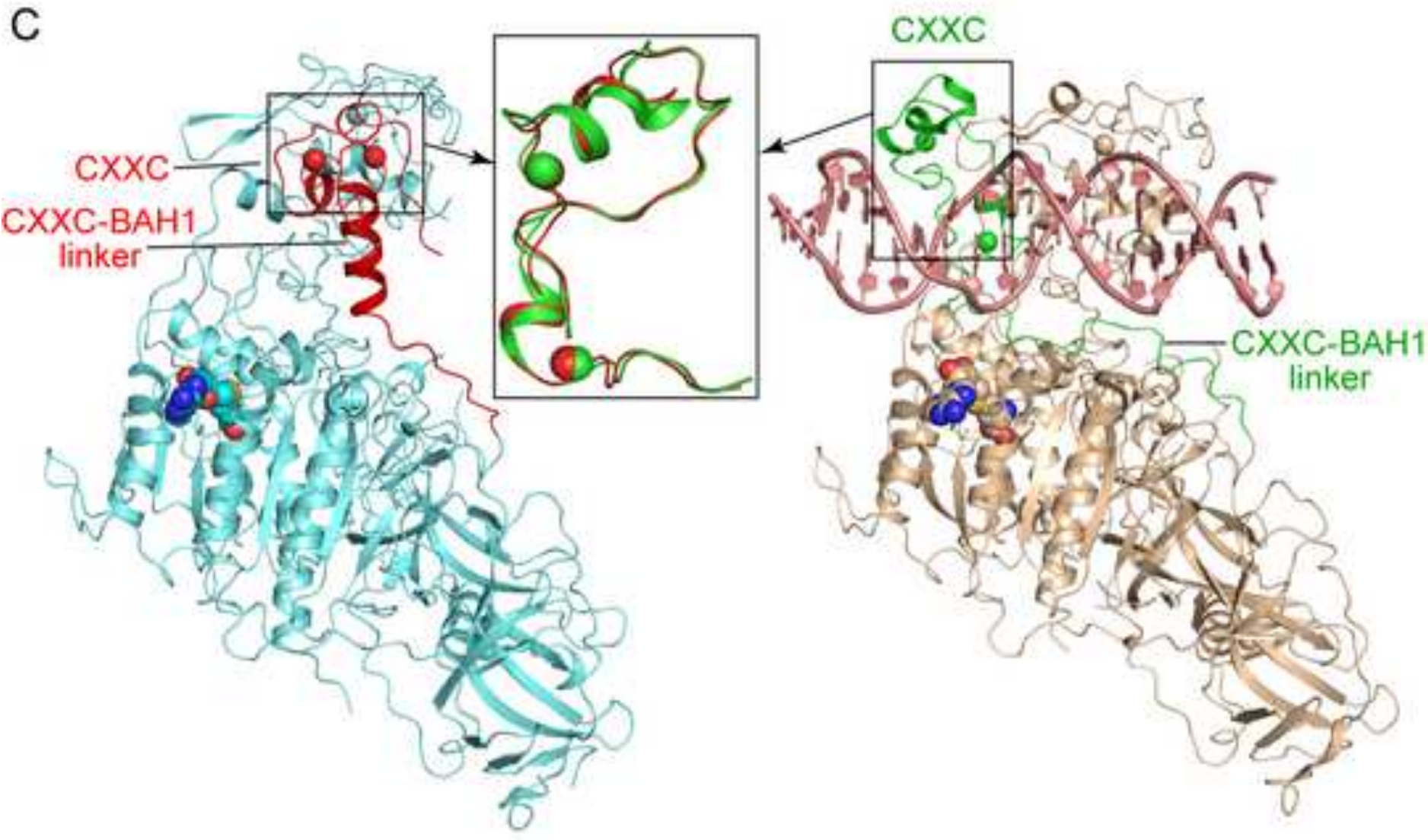

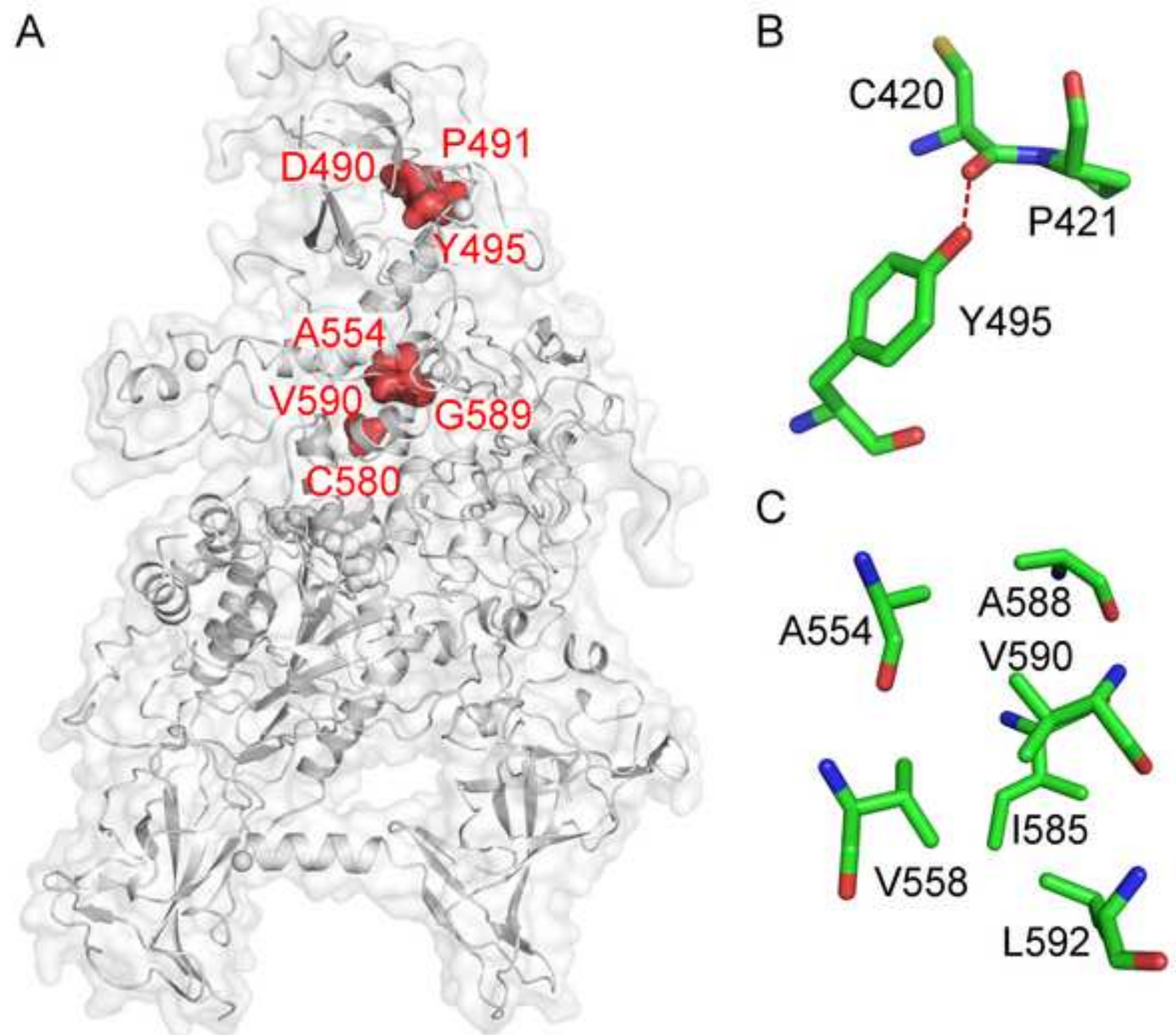


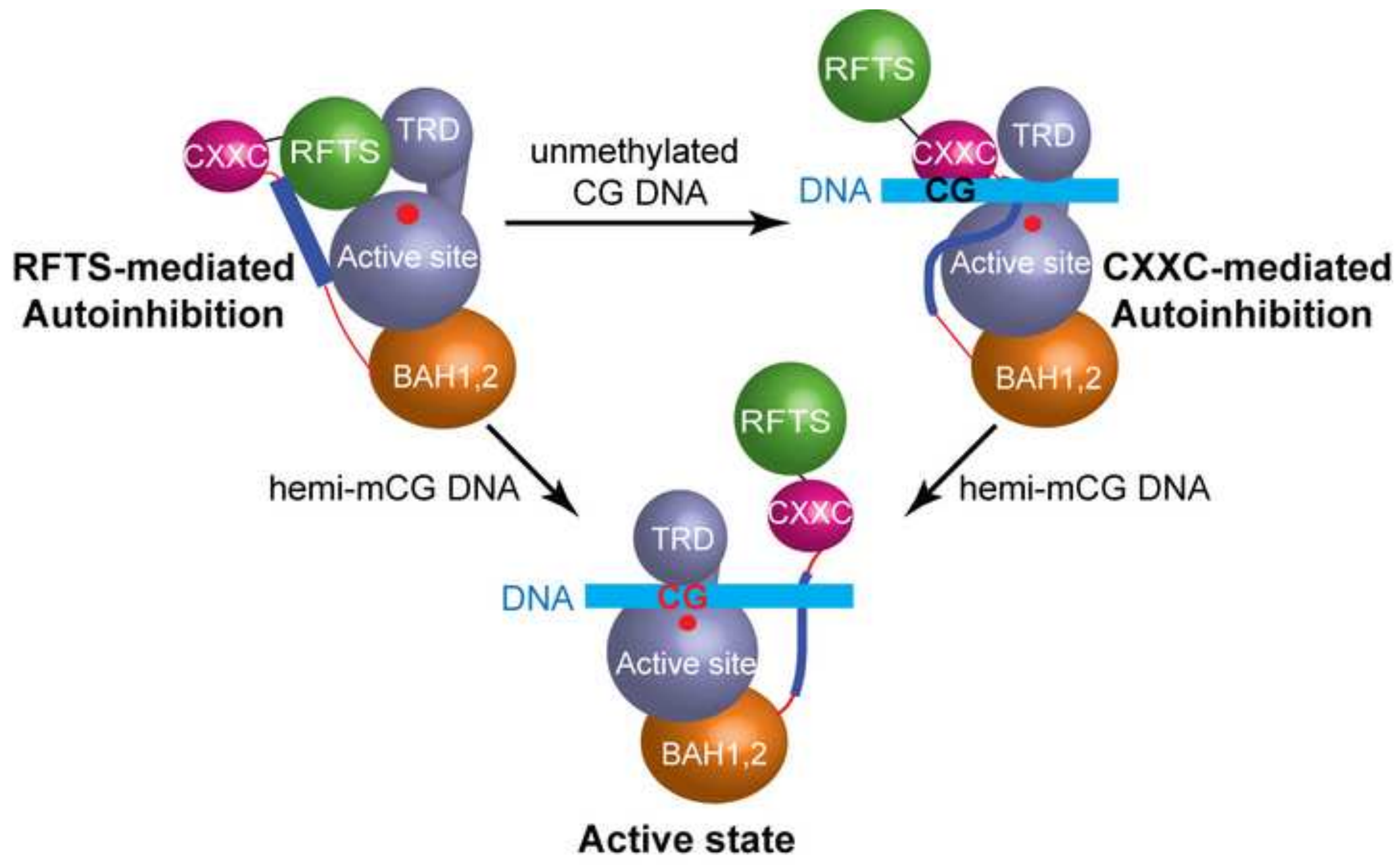



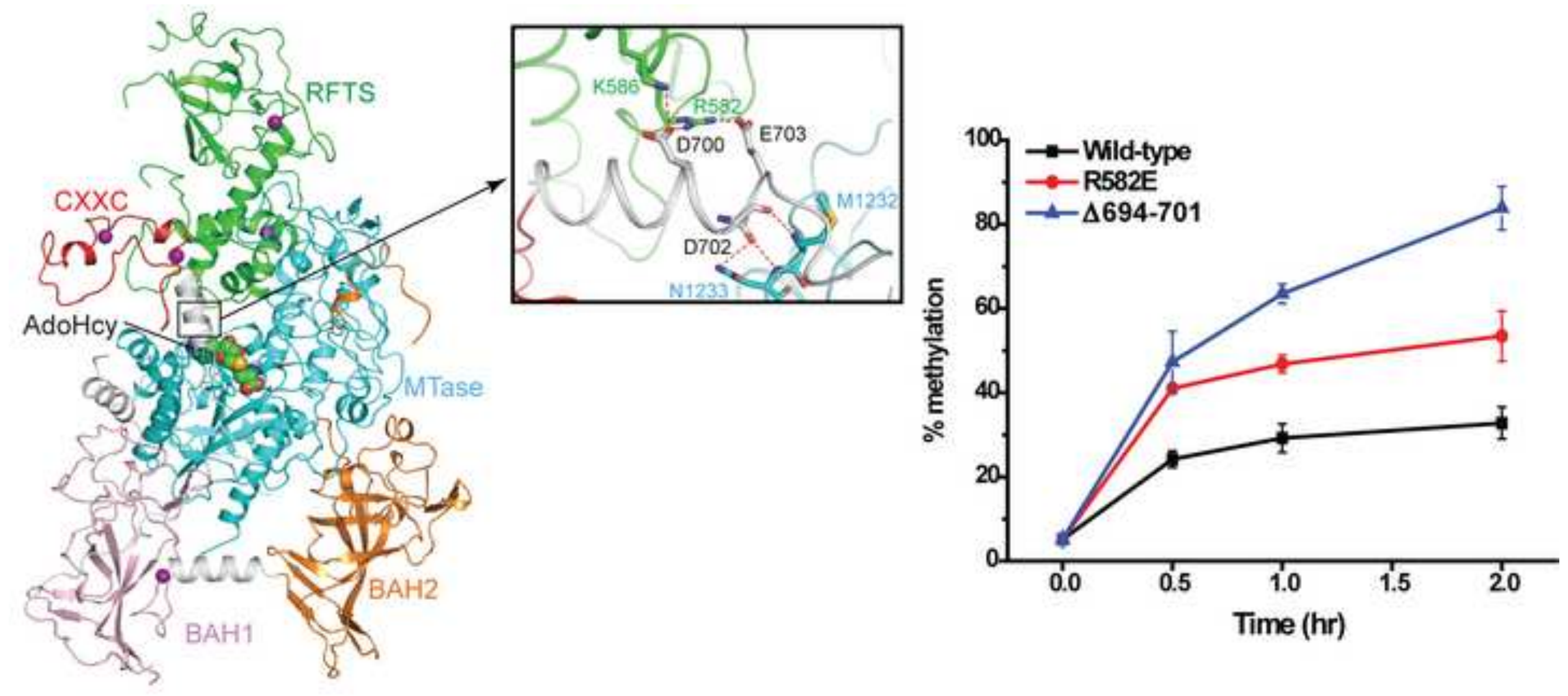